\title{
REVISTAMATĒRIA
}

ISSN 1517-7076 artigo 11746, pp.807-816, 2016

\section{Refino de grão de ligas alumínio- silício com ante-ligas Al-B}

\author{
Grain refinement of silicon-aluminum \\ alloys with master alloys Al-B
}

Alexandre Leão Quadro ${ }^{1}$, Antônio Luís Ribeiro Sabariz ${ }^{2}$, Marco Túlio Raposo ${ }^{1}$

\footnotetext{
${ }^{1}$ Programa de Pós Graduação em Física e Química dos Materiais - FQMat - UFSJ - São João Del Rei

e-mail: aleao@amg-al.com.br; mtraposo@ufsj.edu.br

${ }^{2}$ Departamento de Engenharia Mecânica - DEMEC - UFSJ - São João Del Rei

e-mail: sabariz@ufsj.edu.br
}

\begin{abstract}
RESUMO
Com o objetivo de conhecer a influência das partículas $\mathrm{AlB}_{2}$ e $\mathrm{AlB}_{12}$ na capacidade de refino de grão de uma liga $\mathrm{Al}-4 \mathrm{~B}$ sobre ligas de fundição à base de alumínio-silício, realizou-se testes de refino TP1 da Aluminum Association variando-se as concentrações de $\mathrm{AlB}_{2}$ e $\mathrm{AlB}_{12}$. Para a caracterização química foram utilizadas as técnicas de espectrometria de emissão óptica e espectrometria de energia dispersiva (EDS). O método utilizado para medir os tamanhos de grão foi o do intercepto, através de uma lupa estereoscópica. Os resultados foram comparados com os do refinador convencional Al-5Ti-1B. A liga alumínio-silício utilizada foi a Al$11 \mathrm{Si}-0,1 \mathrm{Mg}$, comum na produção de rodas automotivas. A liga Al-4B com 100\% de partículas $\mathrm{AlB}_{2}$ apresentou a melhor capacidade de refino, proporcionando tamanhos médios de grãos de 0,26 mm na liga Al-11Si0,1Mg, no tempo de amostragem de 5 minutos. A liga Al-4B com 100\% de partículas $\mathrm{AlB}_{12}$ apresentou um resultado intermediário, com tamanhos médios de grãos de $0,43 \mathrm{~mm}$ na liga Al-11Si-0,1Mg, no tempo de 5 minutos. A liga Al-11Si-0,1Mg refinada usando-se o refinador convencional Al-5Ti-1B apresentou tamanho médio de grão de $0,63 \mathrm{~mm}$ após 5 minutos de amostragem, o pior resultado quando comparado com os refinadores $\mathrm{Al}-4 \mathrm{~B}$ com $100 \%$ de partículas $\mathrm{AlB}_{2}$ e $\mathrm{AlB}_{12}$ respectivamente.
\end{abstract}

Palavras-chave: Refino de grão, alumínio, boro.

\begin{abstract}
In order to investigate the influence of $\mathrm{AlB}_{2}$ and $\mathrm{AlB}_{12}$ particles on the grain refining performance of an $\mathrm{Al}-$ 4B master alloy in aluminum-silicon casting alloys, Aluminum Association TP-1 grain size tests were performed for varying concentrations of $\mathrm{AlB}_{2}$ and $\mathrm{AlB}_{12}$. For the chemical characterization, optical emission spectrometry and energy dispersive spectrometry (EDS) were used. Grain sizes were measured by line intercept method using stereoscopic microscope. The results were compared with the conventional grain refiner Al-5Ti-1B. The aluminum-silicon alloy used was the Al-11Si-0.1Mg, common in the production of automotive wheels. The master alloy $\mathrm{Al}-4 \mathrm{~B}$ with $100 \%$ of $\mathrm{AlB}_{2}$ particles showed the best refining performance, providing average grain sizes of $0.26 \mathrm{~mm}$ in the alloy $\mathrm{Al}-11 \mathrm{Si}-0.1 \mathrm{Mg}$ after sampling time of 5 minutes. The alloy $\mathrm{Al}-4 \mathrm{~B}$ with $100 \%$ of $\mathrm{AlB}_{12}$ particles showed an intermediate result, with average sizes of $0.43 \mathrm{~mm}$ in the alloy Al-11Si-0.1Mg after sampling time of 5 minutes. The alloy Al-11Si-0,1Mg refined using the conventional grain refiner Al-5Ti-1B presented average grain size of $0,63 \mathrm{~mm}$ after 5 minutes of sampling time, the worst result compared with the refiners $\mathrm{Al}-4 \mathrm{~B}$ with $100 \%$ of $\mathrm{AlB}_{2}$ and $\mathrm{AlB}_{12}$ particles respectively.
\end{abstract}

Keywords: Grain refine, aluminum, boron.

\section{INTRODUÇÃO}

O refino de grão consiste na obtenção de grãos finos e axiais através da adição controlada de inoculantes no metal líquido, antes da solidificação, proporcionando o mecanismo que é conhecido como nucleação heterogênea [1]. O refino de grão melhora a homogeneidade, as propriedades mecânicas, reduzindo as porosidades, as fraturas a quente e melhora a usinabilidade das peças [2], sendo uma prática antiga e comum na indústria 
do alumínio [3,4]. A Figura 01 mostra uma peça de alumínio sem o tratamento de refino de grão (esquerda) e uma peça com o tratamento de refino de grão (direita).

O método de refino de grão mais utilizado mundialmente é o refino do grão através da adição de ligas refinadoras à base de alumínio, titânio e boro (Al-Ti-B) [1,3,5]. Estas ligas contém dois tipos de partículas. $\mathrm{O}$ primeiro é o diboreto de titânio $\left(\mathrm{TiB}_{2}\right)$, que é um material cerâmico muito estável e inerte, com tamanho entre 1 a $2 \mu \mathrm{m}$, com uma estrutura hexagonal que não se dissolve no alumínio. O segundo é o alumineto de titânio $\left(\mathrm{TiAl}_{3}\right.$ ), com tamanhos na faixa de 30 a $50 \mu \mathrm{m}$ e que se dissolve rapidamente no alumínio fundido. Deste modo, um grande número de partículas de $\mathrm{TiB}_{2}$ está disperso no metal fundido para funcionarem como centros ativos de nucleação durante a solidificação [1]

É bem conhecido que certos elementos químicos podem afetar negativamente a eficiência destas ligas refinadoras à base de $\mathrm{Al}-\mathrm{Ti}-\mathrm{B}$, o que é chamado de envenenamento do refino de grão. O silício é um destes elementos e para ligas de fundição baseadas em alumínio-silício, nota-se que a partir de $2 \%$ a $3 \%$ de silício inicia-se o efeito de envenenamento, que se agrava com o aumento deste teor [1,3,6]. Segundo Qiu, 2007, este efeito de envenenamento ocasionado pelo silício é devido à formação de uma camada de $\mathrm{Ti}_{5} \mathrm{Si}_{3}$ sobre a partícula $\mathrm{TiAl}_{3}$, inibindo as suas propriedades refinadoras [3].

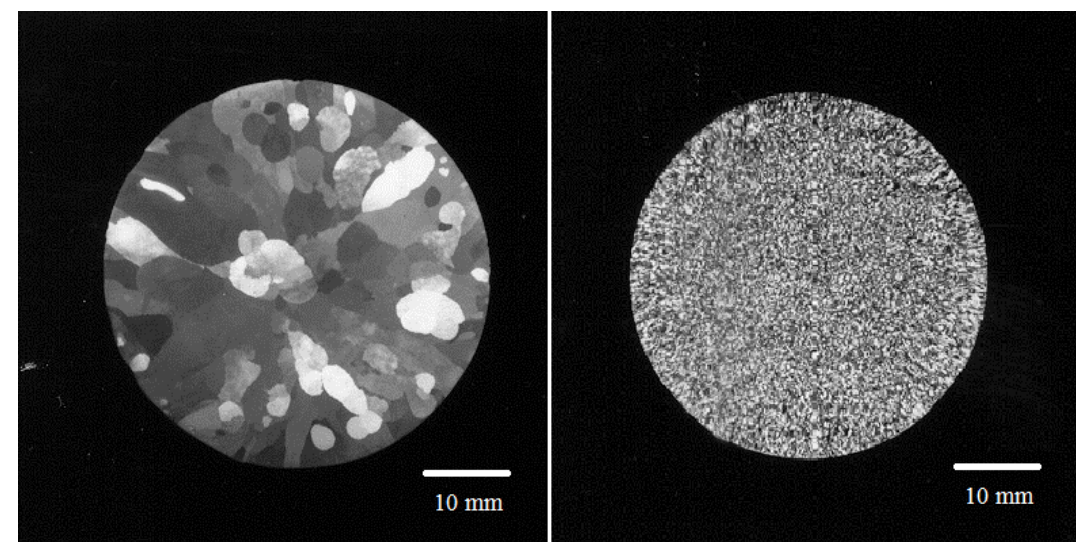

Figura 1: Tratamento de refino de grão. (esquerda: sem tratamento ; direita: com tratamento). (Autoria própria)

Várias tentativas foram feitas para melhorar a resposta ao refino de grão destas ligas Al-Si nas últimas décadas, principalmente porque um tamanho de grão mais fino melhora o preenchimento do molde, resultando em menos porosidades e fraturas a quente [3,7]. Uma maneira utilizada para melhorar este refino de grão nas ligas Al-Si é aumentar a quantidade da adição de ligas refinadoras Al-5Ti-1B [3,8], porém esta não é uma boa solução sob o ponto de vista econômico [3,2].

Algumas novas ligas refinadoras, como as do sistema Al-B, têm sido reportadas como mais efetivas do que os refinadores convencionais Al-Ti-B [3].

Durante a produção destas ligas do sistema Al-B, é possível a formação de dois tipos de partículas: o $\mathrm{AlB}_{2}$ ou o $\mathrm{AlB}_{12}$, dependendo da temperatura de trabalho. Para uma relação molar de 0,1 de Boro/(Alumínio+Boro), ( aproximadamente 4\% de B em peso), entre as temperaturas de $913 \mathrm{~K}$ e $1233 \mathrm{~K}$ a partícula formada é o $\mathrm{AlB}_{2}$ e acima de $1.233 \mathrm{~K}$ a partícula formada é o $\mathrm{AlB}_{12}$, conforme pode ser visto no diagrama de fases da Figura 02. $\mathrm{O} \mathrm{AlB}_{2}$ tem uma estrutura cristalina hexagonal compacta, com parâmetros de rede a $=0,3006 \mathrm{~nm}$ e $\mathrm{c}=0,3252 \mathrm{~nm}$, já o $\mathrm{AlB}_{12}$ tem uma estrutura cristalina tetragonal com parâmetros $\mathrm{a}=1,061 \mathrm{~nm}$ e $\mathrm{b}=1,4238 \mathrm{~nm}$ [9]. Entretanto, não se conhece na literatura o efeito individual de cada partícula sobre a capacidade de refino de uma liga Al-B sobre uma liga Al-Si.

Neste trabalho estudou-se a macro e a microestrutura proporcionada por um refinador Al-4B, com várias proporções entre $\mathrm{AlB}_{2}$ e $\mathrm{AlB}_{12}$ para comparação com as obtidas utilizando-se o refinador convencional Al-5Ti-1B.

A liga de Al-Si utilizada para o estudo foi a Al-11Si-0,1Mg, muito comum na produção de rodas e outros componentes automotivos. Esta é uma liga de difícil refino pelos refinadores convencionais do sistema Al-Ti-B, devido a seu elevado teor de Si. 


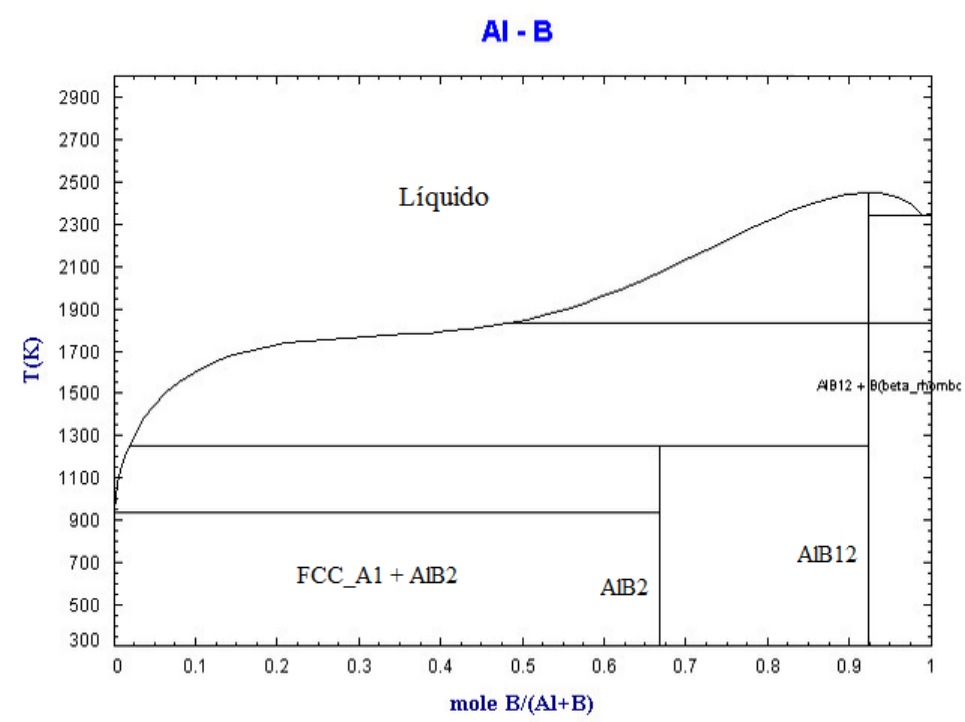

Figura 2: Diagrama de fases Boro-Alumínio [10].

\section{MATERIAIS E MÉTODOS}

\subsection{Produção da liga Al-11Si-0,1Mg}

Para cada teste foi utilizada a liga Al-11Si-0,1Mg, com a composição química objetivada da Tabela 01. Utilizou-se as seguintes matérias-primas para a produção: 8.885 g de alumínio grau P1020; 1.100 g de silício metálico grau metalúrgico; 11 g de magnésio metálico; 27 g de TiAl 10\%. Utilizou-se uma pequena concentração de Ti de 0,030\%, que é desejada quando se utiliza ligas Al-B para o refino de grão [11]. A composição química do TiAl 10\% é mostrada na Tabela 02.

Tabela 1: Composição química objetivada da liga Al-11Si-0,1Mg (\% em peso)

\begin{tabular}{ccccccc}
\hline$\% \mathrm{Si}$ & $\% \mathrm{Mg}$ & $\% \mathrm{Fe}$ & $\% \mathrm{Sr}$ & $\% \mathrm{Ti}$ & $\% \mathrm{Al}$ & Outros \\
\hline 11,0 & 0,10 & 0,10 & $<0,0010$ & 0,030 & Balanço & $0,03 \% \max$ \\
$+/-0,2$ & $+/-0,02$ & $+/-0,03$ & & $+/-0,001$ & & \\
\hline
\end{tabular}

Tabela 02 - Análise química do TiAl 10\% (\% em peso).

\begin{tabular}{cccccc}
\hline$\% \mathrm{Ti}$ & $\% \mathrm{~B}$ & $\% \mathrm{~V}$ & $\% \mathrm{Fe}$ & $\% \mathrm{Si}$ & $\% \mathrm{Al}$ \\
\hline 10,0 & 0,003 & 0,01 & 0,16 & 0,12 & 89,6 \\
\hline
\end{tabular}

Fonte: Certificado do fornecedor (LSM Brasil)

Antes do início dos testes de refino TP1, a liga Al-11Si-0,1Mg foi analisada em um espectrômetro de emissão óptica por centelha, de marca Spectrolab, calibrado com padrões internacionais e quando necessário houve ajuste fino dos teores.

\subsection{Produção das ligas Al-4B}

Foram produzidos dois lotes de ligas Al-4B. O primeiro com a temperatura de trabalho máxima de $1.123 \mathrm{~K}$, objetivando-se a formação de partículas $\mathrm{AlB}_{2}$. O segundo foi produzido com temperatura acima de $1.273 \mathrm{~K}$, objetivando-se a formação de partículas de $\mathrm{AlB}_{12}$.

Utilizou-se como matérias-primas o alumínio primário P1020 e o sal $\mathrm{KBF}_{4}$ na proporção necessária para um teor final de $4 \%$ de B em peso na liga. Foi utilizado um forno de indução de frequência nominal entre 40 a $60 \mathrm{~Hz}$ e a temperatura foi controlada com lingotes de alumínio P1020.

As ligas foram analisadas quimicamente por espectroscopia de emissão óptica por plasma acoplado 
indutivamente (ICP-OES) e microestruturalmente em um microscópio óptico.

\subsection{Teste Padrão de Refino de Grão TP1 - A.A.}

Para a realização dos testes, foi utilizado o procedimento da Aluminum Association - Standard Test Procedure for Aluminum Alloys Grain Refiners TP1 [12].

Este teste consiste da adição de uma quantidade pré-estabelecida do refinador que está em avaliação em $10 \mathrm{~kg}$ da liga líquida (Al-11Si-0,1Mg), em uma temperatura controlada de $680+/-10^{\circ} \mathrm{C}$. As amostras são retiradas com um molde de aço (Figura 03) nos tempos pré-estabelecidos de: tempo 0 (sem adição), 5, 15, 30, 45, 60, 90, 120, 150, 180 e 240 minutos após a adição do refinador. O molde é resfriado em um tanque com uma vazão de água de 3,8 litros/min (Figura 04). Antes da coleta das amostras o banho é agitado por 30 segundos. As amostras resultantes são cortadas a 38 milímetros da base menor do cone, e atacadas por 20 segundos com solução ácida de Poulton (60\% $\mathrm{HCl}$ a $37 \%$; $30 \% \mathrm{HNO}_{3}$ a $65 \%$; $5 \% \mathrm{HF}$ a $50 \%$ e $5 \%$ de água) e clareadas com a solução: $67 \%$ de $\mathrm{HNO}_{3}$ a $65 \%$; $20 \%$ de $\mathrm{HF}$ a $50 \%$ e $13 \%$ de água, para revelar os grãos, que são medidos no plano de corte, sob luz polarizada, através de uma lupa estereoscópica e pelo método do intercepto.

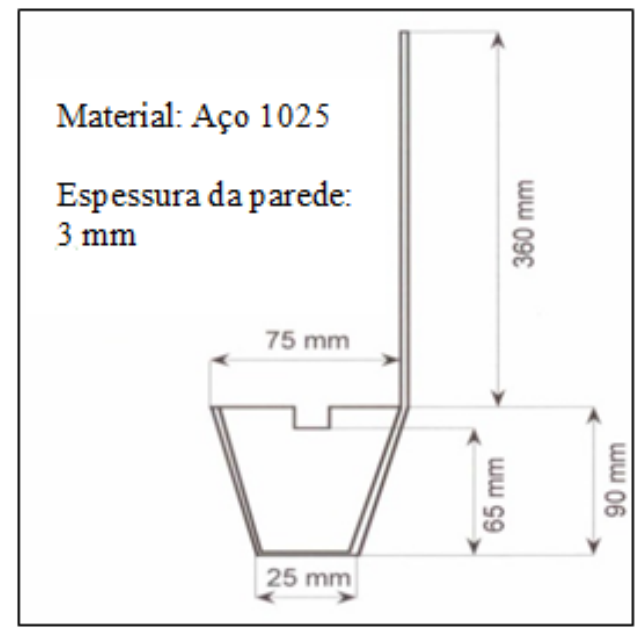

Figura 3: Esquema do molde utilizado para retirar as amostras [12].

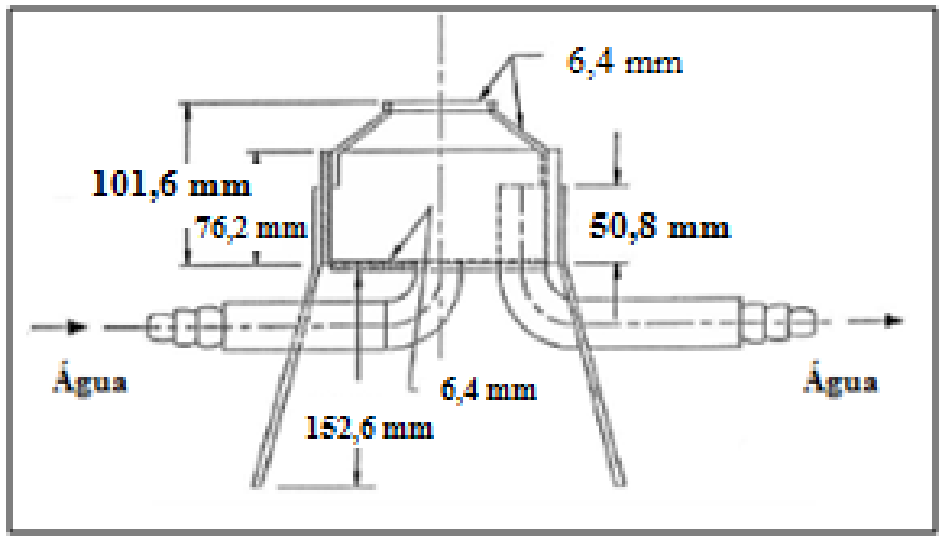

Figura 4: Esquema do tanque de resfriamento utilizado para resfriar o molde [12].

\subsection{Teste Padrão de Refino Alterando $\mathrm{AlB}_{2} / \mathrm{AlB}_{12}$}

Foram realizados 4 testes, todos em triplicata, variando-se a taxa de adição do refinador contendo as partículas $\mathrm{AlB}_{2}$ e $\mathrm{AlB}_{12}$, conforme Tabela 03. 
Tabela 3: Teste de refino realizado com várias proporções de $\mathrm{AlB}_{2} / \mathrm{AlB}_{12}$

\begin{tabular}{ccccc}
\hline Refinador & Teste 1 & Teste 2 & Teste 3 & Teste 4 \\
\hline $\mathrm{Al}-4 \mathrm{~B}$ com $\mathrm{AlB}_{12}$ & $0 \mathrm{~kg} / \mathrm{t}$ & $1 \mathrm{~kg} / \mathrm{t}$ & $3 \mathrm{~kg} / \mathrm{t}$ & $4 \mathrm{~kg} / \mathrm{t}$ \\
$\mathrm{Al}-4 \mathrm{~B}$ com $\mathrm{AlB}_{2}$ & $4 \mathrm{~kg} / \mathrm{t}$ & $3 \mathrm{~kg} / \mathrm{t}$ & $1 \mathrm{~kg} / \mathrm{t}$ & $0 \mathrm{~kg} / \mathrm{t}$ \\
\hline
\end{tabular}

Para efeitos comparativos foi realizado o teste de refino (também em triplicata) com uma taxa de adição de $5 \mathrm{~kg} / \mathrm{t}$ de para o refinador convencional Al-5Ti-1B de teor comercial. Esta taxa de adição foi escolhida para que a \%Ti final do banho seja igual à dos testes 1 a $4(\% \mathrm{Ti}=0,030 \%)$.

\subsection{Tratamento Estatístico dos Resultados}

Foi utilizada a distribuição t de Student, com 90\% de confiança, para a determinação dos intervalos descritos nas Tabelas 05, 06 e 07.

\section{RESULTADOS}

\subsection{Produção da liga Al-4B}

As análises químicas das ligas Al-4B ficaram dentro do esperado, Tabela 04. As pequenas variações entre os dois lotes não afetaram os resultados dos testes.

Fotos da microestrutura dos dois lotes podem ser observadas nas Figuras 05 e 06 . As partículas de $\mathrm{AlB}_{12}$ apresentaram um tamanho maior do que as partículas de $\mathrm{AlB}_{2}$, pois demandam maiores tempos e temperaturas de reação para a sua produção.

Tabela 4 : Análise química Al-4B com $\mathrm{AlB}_{2}$ e $\mathrm{AlB}_{12}$ (\% em peso).

\begin{tabular}{lllll}
\hline $\mathrm{Al}-4 \mathrm{~B}$ & $\% \mathrm{~B}$ & $\% \mathrm{Fe}$ & \%Si & \%Ti \\
\hline $\mathrm{Com} \mathrm{AlB}$ & 4,00 & 0,28 & 0,19 & 0,05 \\
\hline $\mathrm{Com} \mathrm{AlB}$ & 4,07 & 0,22 & 0,16 & 0,05 \\
\hline
\end{tabular}

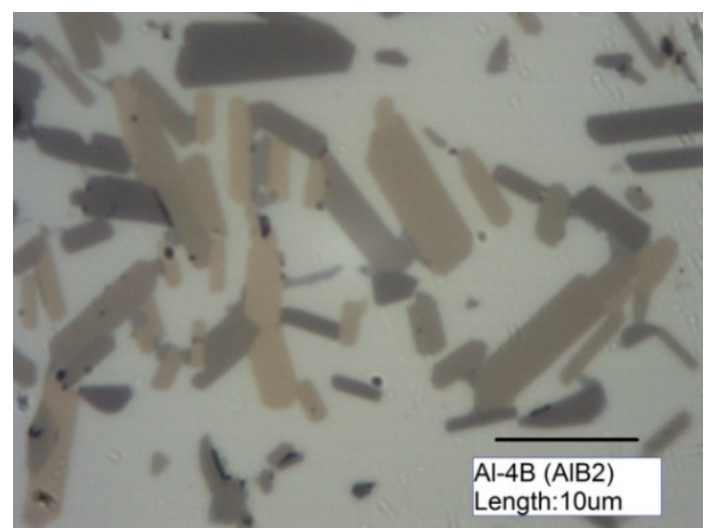

Figura 5: Partículas de $\mathrm{AlB}_{2}$ na microestrutura da liga $\mathrm{Al}-4 \mathrm{~B}(1.000 \mathrm{x})$

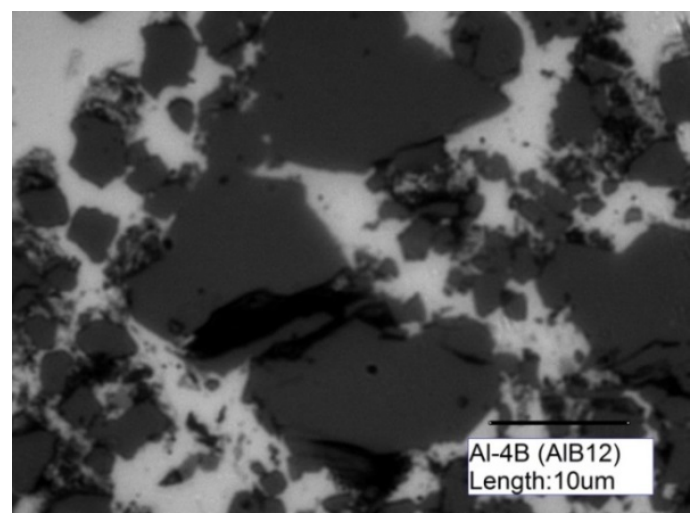

Figura 6: Partículas de $\mathrm{AlB}_{12}$ na microestrutura da liga $\mathrm{Al}-4 \mathrm{~B}(1.000 \mathrm{x})$ 


\subsection{Teste de refino de grão}

$\mathrm{O}$ teste de refino realizado com $4 \mathrm{~kg} / \mathrm{t}$ de $\mathrm{Al}-4 \mathrm{~B}$ com partículas de $\mathrm{AlB}_{2}$ (teste 1 ) apresentou o melhor refino de grão sobre a liga Al-11Si-0,1Mg, com um tamanho médio de grãos de 0,26 mm para o tempo de amostragem de 5 minutos (Tabela 05 e Figura 07).

À medida que se reduz a quantidade de partículas de $\mathrm{AlB}_{2}$ e aumenta-se a quantidade de partículas $\mathrm{AlB}_{12}$ há uma deterioração da capacidade de refino do refinador $\mathrm{Al}-4 \mathrm{~B}$, variando de $0,26 \mathrm{~mm}$ com $100 \%$ de Al-4B com partículas de $\mathrm{AlB}_{2}$ até $0,43 \mathrm{~mm}$ com $100 \%$ de $\mathrm{Al}-4 \mathrm{~B}$ com partículas de $\mathrm{AlB}_{12}$. No gráfico da Figura 08 é mostrado a variação dos tamanhos de grão dos testes 1 ao 4, no tempo de amostragem de 5 minutos, conforme Tabela 07.

O teste que apresentou o pior resultado foi o realizado com uma taxa de adição de $5 \mathrm{~kg} / \mathrm{t}$ do refinador convencional Al-5Ti-1B, obtendo-se um tamanho médio de grão de 0,626 mm no tempo de 5 minutos. Fotos destes grãos, após ataque químico e sob luz polarizada, podem vistas nas Figuras 09 e 10.

Tabela 5: Tamanho de grão obtidos nos testes de refino com Al-4B.

\begin{tabular}{|c|c|c|c|c|}
\hline $\begin{array}{c}\text { Tempo } \\
\text { (min) }\end{array}$ & $\begin{array}{c}\text { Teste } 1 \\
0 \mathrm{~kg} / \mathrm{t} \mathrm{c} / \mathrm{AlB}_{12} \\
4 \mathrm{~kg} / \mathrm{t} \mathrm{c} / \mathrm{AlB}_{2} \\
\end{array}$ & $\begin{array}{c}\text { Teste } 2 \\
1 \mathrm{~kg} / \mathrm{t} \mathrm{c} / \mathrm{AlB}_{12} \\
3 \mathrm{~kg} / \mathrm{t} \mathrm{c} / \mathrm{AlB}_{2} \\
\end{array}$ & $\begin{array}{c}\text { Teste } 3 \\
3 \mathrm{~kg} / \mathrm{t} \mathrm{c} / \mathrm{AlB}_{12} \\
1 \mathrm{~kg} / \mathrm{t} \mathrm{c} / \mathrm{AlB}_{2} \\
\end{array}$ & $\begin{array}{c}\text { Teste } 4 \\
4 \mathrm{~kg} / \mathrm{t} \mathrm{c} / \mathrm{AlB}_{12} \\
0 \mathrm{~kg} / \mathrm{t} \mathrm{c} / \mathrm{AlB}_{2} \\
\end{array}$ \\
\hline 0 & $1,0 \pm 0,2$ & $1,0 \pm 0,1$ & $1,1 \pm 0,2$ & $0,94 \pm 0,1$ \\
\hline 5 & $0,26 \pm 0,04$ & $0,30 \pm 0,01$ & $0,36 \pm 0,08$ & $0,43 \pm 0,10$ \\
\hline 15 & $0,29 \pm 0,04$ & $0,34 \pm 0,09$ & $0,34 \pm 0,06$ & $0,46 \pm 0,20$ \\
\hline 30 & $0,31 \pm 0,06$ & $0,35 \pm 0,05$ & $0,39 \pm 0,09$ & $0,49 \pm 0,10$ \\
\hline 45 & $0,33 \pm 0,07$ & $0,40 \pm 0,10$ & $0,44 \pm 0,20$ & $0,54 \pm 0,08$ \\
\hline 60 & $0,38 \pm 0,02$ & $0,39 \pm 0,09$ & $0,45 \pm 0,07$ & $0,55 \pm 0,04$ \\
\hline 90 & $0,35 \pm 0,04$ & $0,41 \pm 0,15$ & $0,48 \pm 0,15$ & $0,51 \pm 0,08$ \\
\hline 120 & $0,35 \pm 0,03$ & $0,43 \pm 0,08$ & $0,47 \pm 0,06$ & $0,56 \pm 0,18$ \\
\hline 150 & $0,40 \pm 0,08$ & $0,49 \pm 0,10$ & $0,50 \pm 0,11$ & $0,54 \pm 0,07$ \\
\hline 180 & $0,38 \pm 0,04$ & $0,44 \pm 0,13$ & $0,50 \pm 0,11$ & $0,58 \pm 0,10$ \\
\hline 240 & $0,44 \pm 0,11$ & $0,50 \pm 0,09$ & $0,52 \pm 0,12$ & $0,57 \pm 0,04$ \\
\hline
\end{tabular}

Tabela 6: Tamanho de grão obtidos no teste de refino com Al-5Ti-1B.

\begin{tabular}{cc} 
Tamanho de grão obtidos no teste de refino com Al-5Ti-1B. & (mm) \\
\hline $\begin{array}{c}\text { Tempo } \\
(\text { min })\end{array}$ & $\begin{array}{c}\text { Al-5\%Ti-1\%B } \\
5 \mathrm{~kg} / \mathrm{t}\end{array}$ \\
\hline 0 & $1,01 \pm 0,08$ \\
\hline 5 & $0,63 \pm 0,10$ \\
\hline 15 & $0,71 \pm 0,05$ \\
\hline 30 & $0,73 \pm 0,17$ \\
\hline 45 & $0,71 \pm 0,15$ \\
\hline 60 & $0,75 \pm 0,13$ \\
\hline 90 & $0,78 \pm 0,08$ \\
\hline 120 & $0,78 \pm 0,12$ \\
\hline 150 & $0,82 \pm 0,05$ \\
\hline 180 & $0,76 \pm 0,08$ \\
\hline 240 & $0,79 \pm 0,09$
\end{tabular}

Tabela 7: Tamanhos de grão médios para o tempo de amostra de 5 minutos.

\begin{tabular}{lccc}
\hline & $\begin{array}{c}\text { Al-4B com AlB } 12 \\
(\mathrm{Kg} / \mathrm{t})\end{array}$ & $\begin{array}{c}\text { Al-4B com AlB } \\
(\mathrm{Kg} / \mathrm{t})\end{array}$ & $\begin{array}{c}\text { Tamanho de Grão } \\
(\mathrm{mm})\end{array}$ \\
\hline Teste 1 & 0 & 4 & $0,26 \pm 0,04$ \\
\hline Teste 2 & 1 & 3 & $0,30 \pm 0,01$ \\
\hline Teste 3 & 3 & 1 & $0,36 \pm 0,08$ \\
\hline Teste 4 & 4 & 0 & $0,43 \pm 0,10$ \\
\hline
\end{tabular}




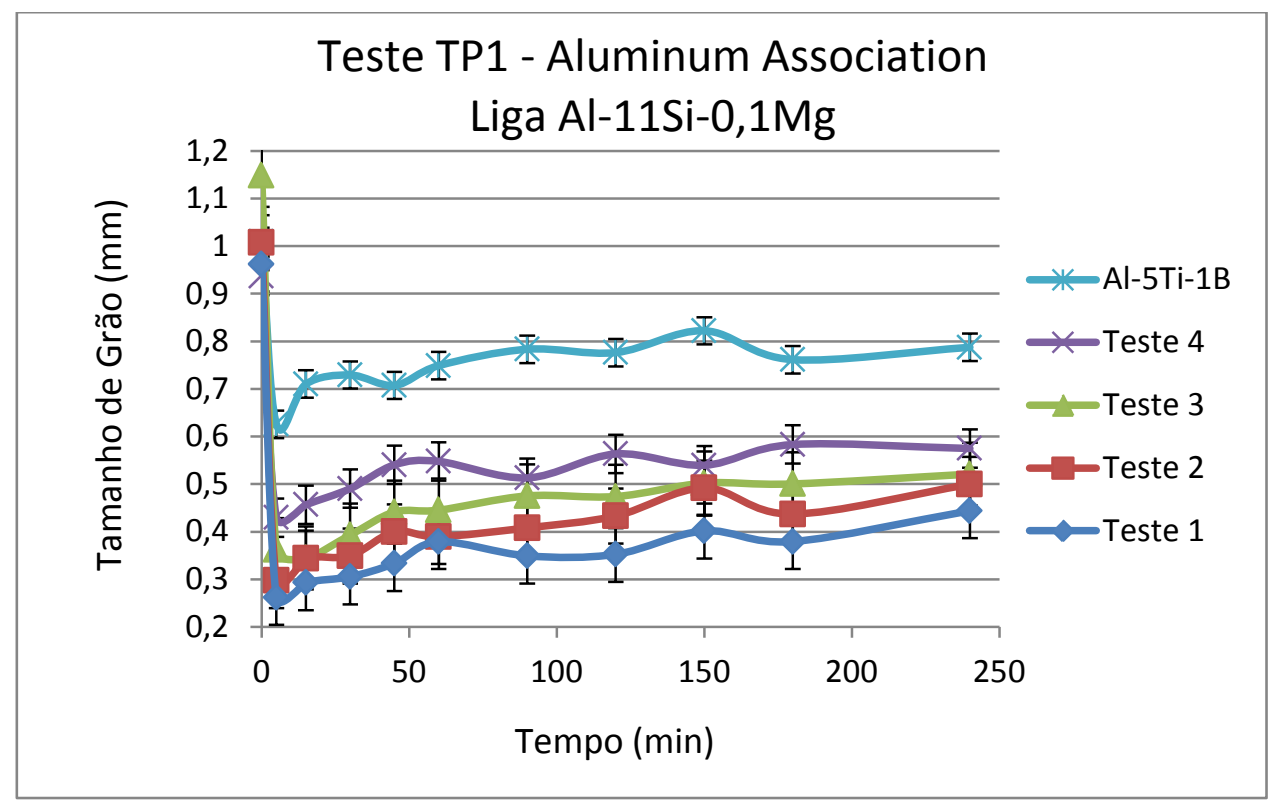

Figura 7: Tamanhos médios de grão para os testes realizados.

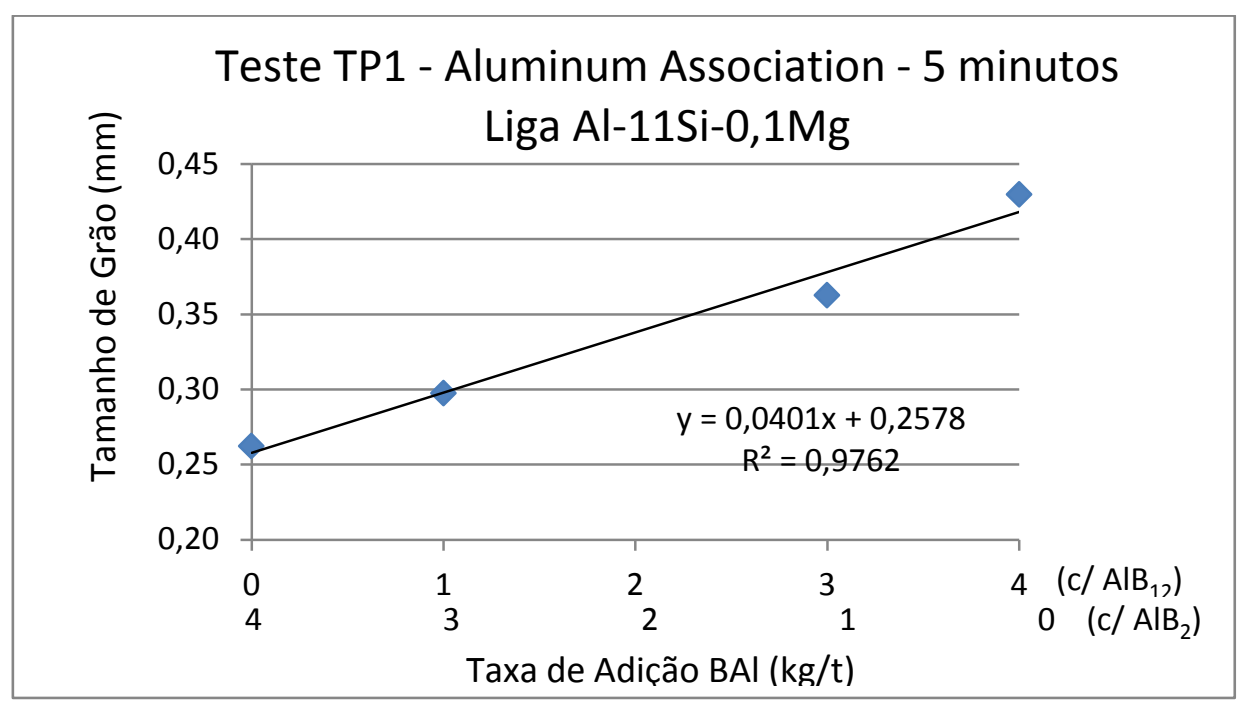

Figura 8: Tamanho de grão para o tempo de 5 minutos
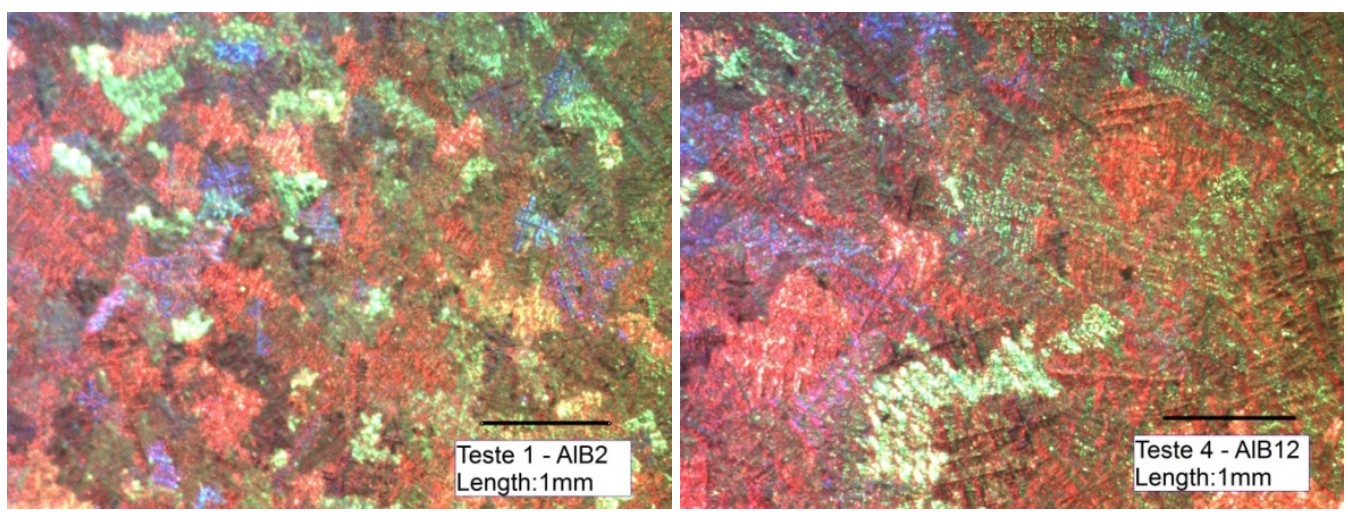

Figura 9: Fotos dos grãos. Teste 1 (4 kg de Al-4B com AlB2) e Teste 4 (4 kg de Al-4B com AlB12), 5 minutos 


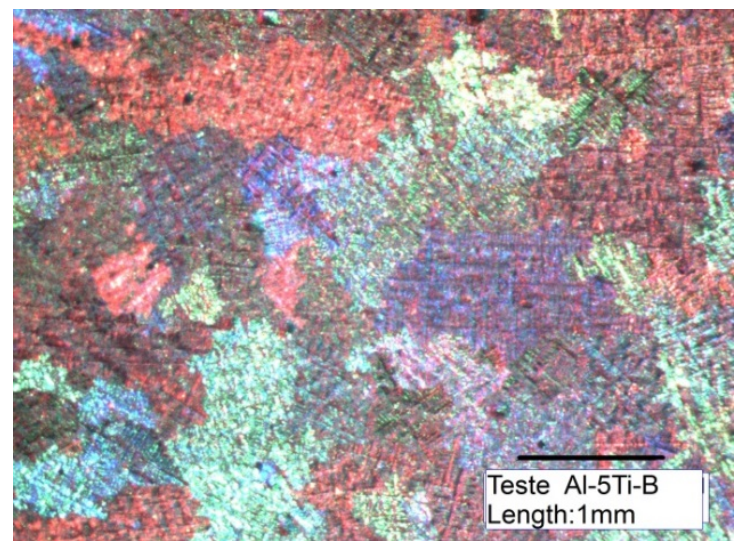

Figura 10: Fotos dos grãos do teste com 5 kg/t de Al-5Ti-1B, 5 minutos

\subsection{EDS da partícula de AlB $_{12}$}

Pela análise EDS, foi possível identificar um anel de $\mathrm{TiB}_{2}$ sobre as partículas de $\mathrm{AlB}_{12}$. Na Figura 11 pode ser observada uma partícula de $\mathrm{AlB}_{12}$, referente ao teste 4 e tempo de amostragem de 5 minutos. O equipamento utilizado foi um microscópio eletrônico de varredura, modelo TM 3000 do fabricante Hitachi. A voltagem de aceleração foi de $15.000 \mathrm{~V}$.

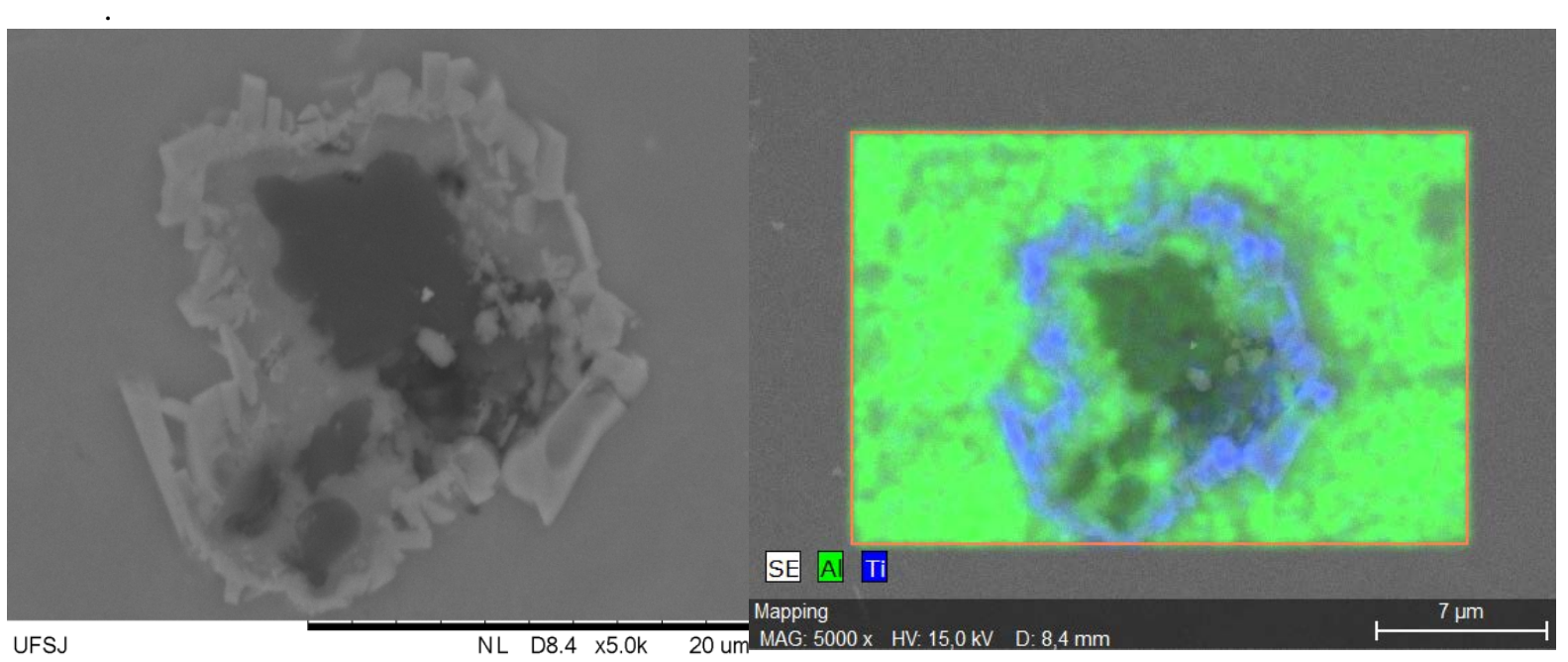

Figura 11: Partícula de $\mathrm{AlB}_{12}$ (núcleo) envolta por um anel de $\mathrm{TiB}_{2}$ (5.000x)

\section{DISCUSSÃO}

Segundo Kalih 2014 [13], quando uma ante-liga de Al-B contendo partículas de $\mathrm{AlB}_{12}$ ou $\mathrm{AlB}_{2}$ é adicionada a um banho de alumínio ocorre a seguinte sequência de eventos:

1 - Liberação das partículas de $\mathrm{AlB}_{12}$ ou $\mathrm{AlB}_{2}$ no banho, seguido de sua dissolução

$$
\text { Ante-liga } \mathrm{Al}-\mathrm{B}=>\mathrm{AlB}_{12(\mathrm{~s})} / \mathrm{AlB}_{2(\mathrm{~s})}+\mathrm{Al}_{(\mathrm{l})}
$$

A dissolução das partículas é controlada pela transferência de massa através da interface sólido/líquido, devido ao maior ponto de fusão destas partículas em relação ao banho [13].

$$
\begin{aligned}
& \mathrm{AlB}_{12(\mathrm{~s})}=>\mathrm{Al}_{(\mathrm{l})}+12[\mathrm{~B}] \\
& \mathrm{AlB}_{2(\mathrm{~s})}=>\mathrm{Al}_{(\mathrm{l})}+2[\mathrm{~B}]
\end{aligned}
$$

2 - Transferência de massa dos solutos seguido de reação química 
Através da transferência de massa do B liberado pela ante-liga Al-B e dos elementos de transição presentes no banho, diboretos são formados através de reação química. Resultados experimentais mostram que esta reação inicial é rápida e uma camada de diboretos é formada próximo à interface das partículas de $\mathrm{AlB}_{\mathrm{X}}$ ainda não dissolvidas [13].

\subsection{Difusão dos elementos através da camada de diboretos}

Para que reação prossiga, agora é necessário a difusão do B através desta camada sólida de diboretos formada ao redor das partículas $\mathrm{AlB}_{\mathrm{X}}$, o que reduz a cinética global da reação e aumenta o tempo de residência das partículas de $\mathrm{AlB}_{\mathrm{X}}[14]$.

Portanto, as partículas de $\mathrm{AlB}_{12}$ ou $\mathrm{AlB}_{2}$ dissolvem no banho fornecendo boro livre para a reação com os elementos de transição Ti, V, Zr e Cr, formando diboretos destes elementos [14]. Como neste trabalho foram adicionados $0,03 \%$ de Ti, necessários para o refino de grão com as anteligas do sistema Al-B [11], pode ser observado pela análise EDS um anel de $\mathrm{TiB}_{2}$ ao redor de uma partícula de $\mathrm{AlB}_{12}$ parcialmente dissolvida, Figura 11.

Como a formação da camada de $\mathrm{TiB}_{2}$ sobre as partícula de $\mathrm{AlB}_{\mathrm{X}}$ ocorre rapidamente e antes da solidificação, podemos considerar que, quando existe Ti disponível, a partícula nucleante é o $\mathrm{TiB}_{2}$ e não o $\mathrm{AlB}_{2}$ ou o $\mathrm{AlB}_{12}$. Esta constatação explica o fato de que uma partícula de $\mathrm{AlB}_{12}$, com estrutura cristalina bastante diferente da estrutura do $\mathrm{AlB}_{2}$ possa atuar como nucleante do $\alpha$-Al, embora que de forma indireta.

Uma das mais importantes questões a ser considerada no entendimento do refino de grão é a potencia do substrato. A energia interfacial entre o substrato e o sólido é crítica e deve ser a menor possível. Para uma baixa energia interfacial, os dois sólidos devem ser coerentes ou parcialmente coerentes, o que leva a uma relação de orientação entre as duas fases. Segundo Zhang, 2005 [15], se o valor da diferença entre os parâmetros cristalinos for menor ou igual a 10\% é possível que exista uma relação de orientação entre os dois cristais.

Os principais nucleantes conhecidos na literatura para o $\alpha$-Al são: $\mathrm{TiAl}_{3}, \mathrm{TiB}_{2}, \mathrm{AlB}_{2}$ e o TiC. As partículas de $\mathrm{TiB}_{2}$ e $\mathrm{AlB}_{2}$ apresentam estrutura hexagonal compacta e parâmetros cristalinos muito semelhantes. Segundo Easton, 1999 [5] a diferença dos parâmetros cristalinos entre os planos compactos (111) $\mathrm{Al} /(0001)_{(\mathrm{Al}}$ ou $\mathrm{Ti)B}$ é de apenas 3.5\% para o $\mathrm{AlB}_{2}$ e de 4,3\% para o $\mathrm{TiB}_{2}$, indicando que ambas partículas podem ser consideradas boas nucleantes para o $\alpha$-Al [5].

Nos testes realizados, ficou evidenciado que um aumento da relação $\mathrm{AlB}_{12} / \mathrm{AlB}_{2}$ reduz a capacidade de refino da liga Al-4B. Esta redução pode ser explicada pelo fato das partículas de $\mathrm{AlB}_{2}$ serem menores que as de $\mathrm{AlB}_{12}$ [14], e que um aumento na proporção de $\mathrm{AlB}_{12}$ resultaria em menos núcleos ativos para a nucleação. Porém é necessário um trabalho mais criterioso de caracterização da fração volumétrica e diâmetro médio de cada uma das partículas de $\mathrm{AlB}_{\mathrm{X}}$ para confirmar esta suposição.

Segundo o mecanismo proposto por QIU em 2007 [3], as partículas de $\mathrm{TiAl}_{3}$ presentes nas ligas refinadoras do sistema Al-5Ti-1B são envolvidas por uma camada de $\mathrm{Ti}_{5} \mathrm{Si}_{3}$, que inibe a sua função de refino de grão. $\mathrm{O} \mathrm{Ti}_{5} \mathrm{Si}_{3}$ não têm a capacidade de envolver o outro tipo de partícula presente na liga Al-5Ti-B, o TiB ${ }_{2}$, explicando porque um aumento na quantidade adicionada de Al-5Ti-B melhora o refino de grão nas ligas AlSi [3].

Ainda segundo Qiu 2007, este mecanismo explica o fato das ligas do sistema Al-B serem refinadores mais efetivos que o Al-5Ti-B. A relação entre $\mathrm{AlB}_{2}$ ou $\mathrm{TiB}_{2}$ e o $\mathrm{TiAl}_{3}$ é muito maior nestas ligas do sistema Al-B do que as do Al-5Ti-1B. Desta forma, para um mesmo nível de adição, mais partículas que não são afetadas pelo $\mathrm{Ti}_{5} \mathrm{Si}_{3}$ serão adicionadas ao banho utilizando-se liga Al-4B, proporcionando um melhor refino de grão [3].

\section{CONCLUSÕES}

- Ligas de sistema Al-4B que contenham partículas $\mathrm{AlB}_{2}$ em sua estrutura, são refinadores de grão mais efetivos para as ligas Al-11Si-0,1Mg, apresentando tamanho médio de grão de 0,26 mm para uma adição de 4 $\mathrm{kg} / \mathrm{t}$ e tempo de amostragem de 5 minutos.

- À medida que se reduz a quantidade de partículas de $\mathrm{AlB}_{2}$ e aumenta-se a quantidade de partículas $\mathrm{AlB}_{12}$ ocorre uma redução na capacidade de refino da liga Al-4B. Esta redução é linear, e o tamanho médio de grão varia de 0,26 $\mathrm{mm}$ a $0,43 \mathrm{~mm}$, para tempos de amostragem de 5 minutos.

- A liga convencional Al-5Ti-1B apresentou o pior resultado de refino de grão, com um tamanho médio de grão na liga Al-11Si-0,1Mg de 0,626 mm, para o tempo de amostragem de 5 minutos. 


\section{AGRADECIMENTOS}

Os autores agradecem à LSM Brasil SA / AMG Advanced Metallurgical Group por disponibilizar os equipamentos e matérias primas e à Universidade Federal de São João Del Rei por disponibilizar o MEV/EDS para caracterização da liga.

\section{BIBLIOGRAFIA}

[1] ARANGO, J. M. R., MARTORANO, M. A., "Efeitos da adição do inoculante Al-3\%Ti-1\%B às ligas do sistema Al-Si”, Tecnologia Metalurgia Materiais e Mineração, v.9, pp. 71-79, 2012.

[2] BIROL, Y. “Grain refinement and modification of Al-Si foundry alloys with B and Sr additions”, Materials Science and Technology, v.30, p.p. 1154-1161, 2014.

[3] QIU, D., TAYLOR J. A., ZHANG, M. X., et al., "A mechanism for the poisoning effect of silicon on the grain refinement of Al-Si alloys”, Acta Materialia, v. 55, p.p. 1447-1456, 2007.

[4] APELIAN, D., SIGWORTH, G. K., WHALER, K.R., “Assessment of grain refining and modification of AlSi foundry alloys by thermal analysis”, AFS Transactions, v. 161, p.p. 297-307, 1984.

[5] EASTON, M., STJOHN, D., “Grain refinement of aluminum alloys: Part I. The nucleant and solute paradigms - A review of the literature”, Metallurgical and Materials Transactions A, v. 30, pp. 1613-1623, 1999.

[6] BIROL, Y., “Impact of grain size on mechanical properties of AlSi7Mg0.3 alloy”, Materials Science \& Engineering; v.559, pp. 394-400. 2012

[7] QUESTED, T. E. “Understanding mechanisms of grain refinement of aluminium alloys by inoculation”, Materials Science and Technology, v.20, pp. 1357-1369, 2004.

[8] CHEN, Z., WANG, T., GAO L., et al., "Grain refinement and tensile properties improvements of alluminum foundry alloys by inoculation with Al-B master alloys”, Material Science and Engineering A, v. 553, p.p. 32-36, 2012.

[9] KAYIKCI, R., KURTULUS, O., GURBUZ, R., "The formation and growth behavior of aluminum boride crystals in Al-B alloys”, Solid State Phenomena, v. 144, p.p. 140-144, 2008.

[10] CENTER FOR RESEARCH IN COMPUTATIONAL THERMOCHEMISTRY, http:// www. crct. polymtl.ca/fact/Documentation/SGTE, acessado em outubro de 2015.

[11] SIGWORTH, G. K., KUHN, T. A., “Grain refinement of aluminum casting alloys”, AFS Transactions, paper 07-067 (02), p.p. 1-12, 2007

[12] ALUMINUM ASSOCIATION, "Standard Test Procedure for Aluminum Alloy Grain Refiners - TP1", 2012.

[13] KHALIQ, A., RHAMDHANI, M. A., BROOKS G.A., et al., "Removal of Vanadium from Molten Aluminum - Part I. Analyses of $\mathrm{VB}_{2}$ formation”, Metallurgical and Materials Transactions B, v. 45B, p.p. 752-768, 2014.

[14] KHALIQ, A., RHAMDHANI, M. A., BROOKS G.A., et al., “Analysis of Boron Treatment using AlB2 and AlB12 based Master alloy”, Light Metals, pp. 963-968, 2014.

[15] ZHANG, M.X., KELLY P.M., EASTON M.A., et al., "Crystallographic Study of Grain Refinement in Aluminum Alloys Using the Edge-to-Edge Matching Model”, Acta Materialia; v. 53, pp. 1427-1438, 2005. 